\title{
Some properties of various graphs associated with finite groups*
}

\author{
X. Y. Chen, A. R. Moghaddamfar, and M. Zohourattar
}

Communicated by A. Yu. Olshanskii

\begin{abstract}
A BSTRACT. In this paper we investigate some properties of the power graph and commuting graph associated with a finite group, using their tree-numbers. Among other things, it is shown that the simple group $L_{2}(7)$ can be characterized through the treenumber of its power graph. Moreover, the classification of groups with power-free decomposition is presented. Finally, we obtain an explicit formula concerning the tree-number of commuting graphs associated with the Suzuki simple groups.
\end{abstract}

\section{Notation and definitions}

We will consider finite simple graphs $\Gamma=\left(V_{\Gamma}, E_{\Gamma}\right)$, where $V_{\Gamma} \neq \varnothing$ and $E_{\Gamma}$ are the vertex set and edge set of $\Gamma$, respectively. A clique (or a complete set) in $\Gamma$ is a subset of $V_{\Gamma}$ consisting of pairwise adjacent vertices (we do not require that it be a maximal complete set). Especially, a complete graph is a graph in which the vertex set is a complete set. A coclique (edgeless graph or independent set) in $\Gamma$ is a set of pairwise

${ }^{*}$ This work was done during the first and second authors had a visiting position at the Department of Mathematical Sciences, Kent State University, USA. They would like to thank the hospitality of the Department of Mathematical Sciences of KSU. The first author thanks Cultivation Programme for Young Backbone Teachers in Henan University of Technology, the Project of Henan Province (182102410049), and NSFC (11926330, 11926326, 11971189, 11771356).

2020 MSC: 05C25, 20D05, $20 \mathrm{D} 06$.

Key words and phrases: Power graph, commuting graph, tree-number, simple group. 
nonadjacent vertices. The independence number, denoted by $\alpha(\Gamma)$, is the size of the largest coclique in $\Gamma$.

A spanning tree for a graph $\Gamma$ is a subgraph of $\Gamma$ which is a tree and contains all the vertices of $\Gamma$. The tree-number (or complexity) of a graph $\Gamma$, denoted by $\kappa(\Gamma)$, is the number of spanning trees of $\Gamma(0$ if $\Gamma$ is disconnected), see [57]. The famous Cayley formula shows that the complexity of the complete graph with $n$ vertices is given by $n^{n-2}$ (Cayley's formula).

In this paper, we shall be concerned with some graphs arising from finite groups. Indeed, there are various ways to associate a certain graph to a finite group (for example see [3], [56], [60]). Two well known graphs associated with groups are commuting graphs and power graphs, as defined more precisely below. Let $G$ be a finite group and $X$ a nonempty subset of $G$ :

- The (undirected) power graph $\mathcal{P}(G, X)$, has $X$ as its vertex set with two distinct elements of $X$ joined by an edge when one is a power of the other. The directed power graph $\overrightarrow{\mathcal{P}}(G, X)$ is a directed graph with the set $X$ of vertices and with all edges $(x, y)$ such that $x \neq y$ and $y$ is a power of $x$.

- The commuting graph $\mathcal{C}(G, X)$, has $X$ as its vertex set with two distinct elements of $X$ joined by an edge when they commute in $G$. Clearly, power graph $\mathcal{P}(G, X)$ is a subgraph of $\mathcal{C}(G, X)$. In the case $X=G$, we will simply write $\mathcal{C}(G)$ and $\mathcal{P}(G)$ instead of $\mathcal{C}(G, G)$ and $\mathcal{P}(G, G)$, respectively.

The directed power graph of a group was introduced by Kelarev and Quinn [36]. The definition was formulated so that it applied to semigroups as well. Accordingly, the power graphs of semigroups were first considered in [39], [37] and [38]. It is also explained in the survey [1] that the definition given in [36] covers all undirected graphs as well. This means that the undirected power graphs were also defined in [36] (see [1] for more detailed explanations). All of these papers used only the brief term power graph, even though they covered both directed and undirected power graphs. The investigation of graphs like these is very important, because they have valuable applications presented, for example, in the books [34], [35] and the survey [40].

The power and commuting graphs have been also considered in many other papers, see for instance [2], [4]-[7], [10]-[18], [20,22, 23], [25]-[28], [31][33], [41]-[44], [46]-[48], [50]-[55], [58, 59, 63]. In particular, in [45, Lemma 4.1], it is shown that $\mathcal{P}(G)=\mathcal{C}(G)$ if and only if $G$ is a cyclic group of 
prime power order, or a generalized quaternion 2-group, or a Frobenius group with kernel a cyclic $p$-group and complement a cyclic $q$-group, where $p$ and $q$ are distinct primes. Obviously, when $1 \in X$, the power graph $\mathcal{P}(G, X)$ and the commuting graph $\mathcal{C}(G, X)$ are connected, and we can talk about the complexity of these graphs. For convenience, we put $\kappa_{\mathcal{P}}(G, X)=\kappa(\mathcal{P}(G, X)), \kappa_{\mathcal{P}}(G)=\kappa(\mathcal{P}(G)), \kappa_{\mathcal{C}}(G, X)=\kappa(\mathcal{C}(G, X))$ and $\kappa_{\mathcal{C}}(G)=\kappa(\mathcal{C}(G))$. Also, instead of $\kappa_{\mathcal{P}}(G, X)$ and $\kappa_{\mathcal{C}}(G, X)$, we simply write $\kappa_{\mathcal{P}}(X)$ and $\kappa_{\mathcal{C}}(X)$, if it does not lead to confusion. All groups under discussion in this paper are finite and our group theoretic notation is mostly standard and follows that in [19].

\section{General lemmas}

We first establish some notation which will be used repeatedly in the sequel. Given a graph $\Gamma$, we denote by $\mathbf{A}_{\Gamma}$ and $\mathbf{D}_{\Gamma}$ the adjacency matrix and the diagonal matrix of vertex degrees of $\Gamma$, respectively. The Laplacian matrix of $G$ is defined as $\mathbf{L}_{\Gamma}=\mathbf{D}_{\Gamma}-\mathbf{A}_{\Gamma}$. Clearly, $\mathbf{L}_{\Gamma}$ is a real symmetric matrix and its eigenvalues are nonnegative real numbers. The Laplacian spectrum of $\Gamma$ is

$$
\operatorname{Spec}\left(\mathbf{L}_{\Gamma}\right)=\left(\mu_{1}(\Gamma), \mu_{2}(\Gamma), \ldots, \mu_{n}(\Gamma)\right),
$$

where $\mu_{1}(\Gamma) \geqslant \mu_{2}(\Gamma) \geqslant \cdots \geqslant \mu_{n}(\Gamma)$, are the eigenvalues of $\mathbf{L}_{\Gamma}$ arranged in weakly decreasing order, and $n=|V(\Gamma)|$. Note that, $\mu_{n}(\Gamma)$ is 0 , because each row sum of $\mathbf{L}_{\Gamma}$ is 0 . Instead of $\mathbf{A}_{\Gamma}, \mathbf{L}_{\Gamma}$, and $\mu_{i}(\Gamma)$ we simply write $\mathbf{A}, \mathbf{L}$, and $\mu_{i}$ if it does not lead to confusion.

For a graph with $n$ vertices and Laplacian spectrum

$$
\mu_{1} \geqslant \mu_{2} \geqslant \cdots \geqslant \mu_{n}=0,
$$

it has been proved $[8$, Corollary 6.5$]$ that:

$$
\kappa(\Gamma)=\frac{\mu_{1} \mu_{2} \cdots \mu_{n-1}}{n} .
$$

The vertex-disjoint union of the graphs $\Gamma_{1}$ and $\Gamma_{2}$ is denoted by $\Gamma_{1} \oplus \Gamma_{2}$. Define the join of $\Gamma_{1}$ and $\Gamma_{2}$ to be $\Gamma_{1} \vee \Gamma_{2}=\left(\Gamma_{1}^{c} \oplus \Gamma_{2}^{c}\right)^{c}$. Evidently this is the graph formed from the vertex-disjoint union of the two graphs $\Gamma_{1}, \Gamma_{2}$, by adding edges joining every vertex of $\Gamma_{1}$ to every vertex of $\Gamma_{2}$. Now, one may easily prove the following (see also [49]). 
Lemma 1. Let $\Gamma_{1}$ and $\Gamma_{2}$ be two graphs on disjoint sets with $m$ and $n$ vertices, respectively. If

$$
\operatorname{Spec}\left(\mathbf{L}_{\Gamma_{1}}\right)=\left(\mu_{1}\left(\Gamma_{1}\right), \mu_{2}\left(\Gamma_{1}\right), \ldots, \mu_{m}\left(\Gamma_{1}\right)\right),
$$

and

$$
\operatorname{Spec}\left(\mathbf{L}_{\Gamma_{2}}\right)=\left(\mu_{1}\left(\Gamma_{2}\right), \mu_{2}\left(\Gamma_{2}\right), \ldots, \mu_{n}\left(\Gamma_{2}\right)\right),
$$

then the following hold:

(1) The eigenvalues of Laplacian matrix $\mathbf{L}_{\Gamma_{1} \oplus \Gamma_{2}}$ are:

$$
\mu_{1}\left(\Gamma_{1}\right), \ldots, \mu_{m}\left(\Gamma_{1}\right), \mu_{1}\left(\Gamma_{2}\right), \ldots, \mu_{n}\left(\Gamma_{2}\right) .
$$

(2) The eigenvalues of Laplacian matrix $\mathbf{L}_{\Gamma_{1} \vee \Gamma_{2}}$ are:

$$
\begin{aligned}
m+n, & \mu_{1}\left(\Gamma_{1}\right)+n, \ldots, \mu_{m-1}\left(\Gamma_{1}\right)+n, \\
& \mu_{1}\left(\Gamma_{2}\right)+m, \ldots, \mu_{n-1}\left(\Gamma_{2}\right)+m, 0 .
\end{aligned}
$$

Two Examples. (1) Consider the complete bipartite graph $K_{a, b}=K_{a}^{c} \vee K_{b}^{c}$. Then, by Lemma 1 (2), the eigenvalues of Laplacian matrix $\mathbf{L}_{K_{a}^{c} \vee K_{b}^{c}}$ are:

$$
a+b, \underbrace{b, b, \ldots, b}_{(a-1) \text {-times }}, \underbrace{a, a, \ldots, a}_{(b-1)-\text { times }}, 0 .
$$

Using Eq. (1) we get $\kappa\left(K_{a, b}\right)=b^{a-1} a^{b-1}$.

(2) A graph $\Gamma$ is a split graph if its vertex set can be partitioned into a clique $C$ and an independent set $I$, where $V_{\Gamma}=C \uplus I$ is called a split partition of $\Gamma$ (see [24]). Now, consider the split graph $\Gamma=K_{a} \vee K_{b}^{c}$. Again, by Lemma 1 (2), the eigenvalues of Laplacian matrix $\mathbf{L}_{K_{a} \vee K_{b}^{c}}$ are:

$$
a+b, \underbrace{a+b, a+b, \ldots, a+b}_{(a-1) \text {-times }}, \underbrace{a, a, \ldots, a}_{(b-1) \text {-times }}, 0,
$$

and it follows from Eq. (1) that $\kappa(\Gamma)=(a+b)^{a-1} a^{b-1}$.

Next lemma determines the complete commuting graphs and power graphs.

Lemma 2. Let $G$ be a finite group. Then, we have

(1) The commuting graph $\mathcal{C}(G)$ is complete iff $G$ is an abelian group.

(2) The power graph $\mathcal{P}(G)$ is complete iff $G$ is a cyclic p-group for some prime $p$ (see Theorem 2.12 in [14]). 
An immediate consequence of Lemma 2 is that $\kappa_{\mathcal{P}}\left(\mathbb{Z}_{p^{n}}\right)=p^{n\left(p^{n}-2\right)}$ and if $X \subset G$ is a commuting set, then $\kappa_{\mathcal{C}}(G, X)=|X|^{|X|-2}$. We will use these facts without further references.

Lemma 3. Let $M$ and $N$ be subgroups of a group $G$ such that $M \cap N=1$. Let $x \in M$ and $y \in N$ be two arbitrary nontrivial elements. Then, $x$ and $y$ are nonadjacent in $\mathcal{P}(G)$ as two vertices. In particular, if $m>1$ and $X=\cup_{j=1}^{m} G_{j}$, where $1<G_{j}<G$ and $G_{i} \cap G_{j}=1$ for $i \neq j$, then we have

$$
\mathcal{P}\left(X^{\#}\right)=\bigoplus_{j=1}^{m} \mathcal{P}\left(G_{j}^{\#}\right),
$$

where $X^{\#}=X \backslash\{1\}$ and $G_{j}^{\#}=G_{j} \backslash\{1\}$.

Proof. It is easy to see that $\langle x\rangle \cap\langle y\rangle \subseteq M \cap N=1$, which forces $\langle x\rangle \nsubseteq\langle y\rangle$ and $\langle y\rangle \nsubseteq\langle x\rangle$.

As an immediate consequence of Lemma 3, we obtain

Corollary 1. Let $G$ be a finite group. Then the following hold:

(1) If $G$ has even order, then $\operatorname{Inv}(G)$, the set of involutions of $G$, forms an independent set of $\mathcal{P}(G)$.

(2) Any pair of elements in $G$ with relatively prime orders forms an independent set of $\mathcal{P}(G)$, especially we have $\alpha(\mathcal{P}(G)) \geqslant|\pi(G)|$, where $\pi(G)$ denotes the set of all prime divisors of $|G|$.

A universal vertex is a vertex of a graph that is adjacent to all other vertices of the graph. The following lemma [12, Proposition 4] determines the set of universal vertices of the power graph of $G$, in general case.

Lemma 4. Let $G$ be a finite group and $S$ the set of universal vertices of the power graph $\mathcal{P}(G)$. Suppose that $|S|>1$. Then one of the following occurs:

(a) $G$ is cyclic of prime power order, and $S=G$;

(b) $G$ is cyclic of non-prime-power order $n$, and $S$ consists of the identity and the generators of $G$, so that $|S|=1+\phi(n)$;

(c) $G$ is generalized quaternion, and $S$ contains the identity and the unique involution in $G$, so that $|S|=2$.

Lemma 5. [50, Theorem 3.4] If $H_{1}, H_{2}, \ldots, H_{t}$ are nontrivial subgroups of a group $G$ such that $H_{i} \cap H_{j}=\{1\}$, for each $1 \leqslant i<j \leqslant t$, then we have $\kappa_{\mathcal{P}}(G) \geqslant \kappa_{\mathcal{P}}\left(H_{1}\right) \kappa_{\mathcal{P}}\left(H_{2}\right) \cdots \kappa_{\mathcal{P}}\left(H_{t}\right)$. 
Lemma 6. [50, Lemma 6.1] Let $G$ be a finite nonabelian simple group and let $p$ be a prime dividing the order of $G$. Then $G$ has at least $p^{2}-1$ elements of order $p$, or equivalently, there is at least $p+1$ cyclic subgroups of order $p$ in $G$.

\section{Main results}

\subsection{Power graphs}

We begin with some elementary but useful results of power graphs. Before stating the results, we need to introduce some additional notation. Let $X$ be a nonempty subset of $G^{\#}$, the set of nonidentity elements of $G$. We denote by $1_{X}$ the bipartite graph with partite sets $\{1\}$ and $X$. Let $\phi$ denote Euler's totient function, so that $\phi(n)=\left|\mathbb{Z}_{n}^{\times}\right|$. We will preserve these notation throughout this section.

Lemma 7. Let $G$ be a group and $H$ be a proper subgroup of $G$. If $m$ is the order of an element of $G \backslash H$, then we have

$$
\kappa_{\mathcal{P}}(G) \geqslant(\phi(m)+1)^{\phi(m)-1} \kappa_{\mathcal{P}}(H) .
$$

In particular, $\kappa_{\mathcal{P}}(G) \geqslant \kappa_{\mathcal{P}}(H)$, with equality if and only if $G$ is a Frobenius group with kernel $H$ and complement $C$ of order 2.

Proof. Let $x$ be an element in $G \backslash H$ of order $m$. Set

$$
\Omega_{x}=\{y \mid\langle y\rangle=\langle x\rangle\} \cup\{1\} .
$$

Clearly, $\Omega_{x} \cap H=1$ and $\left|\Omega_{x}\right|=\phi(m)+1$. Note that the induced subgraph $\mathcal{P}\left(G, \Omega_{x}\right)$ is a complete graph, that is $\mathcal{P}\left(G, \Omega_{x}\right)=K_{\phi(m)+1}$. Now, if $T_{\Omega_{x}}$ and $T_{H}$ are two arbitrary spanning trees of $\mathcal{P}\left(G, \Omega_{x}\right)$ and $\mathcal{P}(G, H)$, respectively, then $T_{G}=T_{\Omega_{x}} \cup T_{H} \cup 1_{G \backslash\left(\Omega_{x} \cup H\right)}$ is a spanning tree of $\mathcal{P}(G)$. Thus, by product rule the number of such spanning trees of $\mathcal{P}(G)$ is equal to

$$
\kappa_{\mathcal{P}}\left(\Omega_{x}\right) \cdot \kappa_{\mathcal{P}}(H) \cdot 1=(\phi(m)+1)^{\phi(m)-1} \kappa_{\mathcal{P}}(H) . \quad \text { (by Cayley's formula) }
$$

This shows that the following inequality holds:

$$
\kappa_{\mathcal{P}}(G) \geqslant(\phi(m)+1)^{\phi(m)-1} \kappa_{\mathcal{P}}(H),
$$


as required. Finally, since for each positive integer $m$,

$$
(\phi(m)+1)^{\phi(m)-1} \geqslant 1
$$

it follows that $\kappa_{\mathcal{P}}(G) \geqslant \kappa_{\mathcal{P}}(H)$.

The preceding argument suggests how to construct a spanning tree of $\mathcal{P}(G)$ through a spanning tree of $\mathcal{P}(G, H)$. In fact, if $T_{H}$ is a spanning tree of $\mathcal{P}(G, H)$, then $T_{G}=T_{H} \cup 1_{G \backslash H}$ is a spanning tree of $\mathcal{P}(G)$, which leads again to the inequality $\kappa_{\mathcal{P}}(G) \geqslant \kappa_{\mathcal{P}}(H)$. Moreover, the equality holds if and only if $\mathcal{P}(G, G \backslash H)$ is a null graph and there are no edges between vertices in $G \backslash H$ and $H^{\#}$. We argue under these conditions that $G$ is a Frobenius group with kernel $H$ and complement $C$ of order 2. We first observe that every element in $G \backslash H$ is an involution. Also, for all $x \in G \backslash H$ and $h \in H, x h \in G \backslash H$ and so $(x h)^{2}=1$, or equivalently $x^{-1} h x=h^{-1}$. This shows that $H$ is a normal subgroup of $G$ and the cyclic subgroup $C=\langle x\rangle$ of order 2 acts on $H$ by conjugation which induces a fixed-point-free automorphism of $H$. Hence, $G=H C$ is a Frobenius group with kernel $H$ and complement $C$, as required.

A group $G$ from a class $\mathcal{F}$ is said to be recognizable in $\mathcal{F}$ by $\kappa_{\mathcal{P}}(G)$ (shortly, $\kappa_{\mathcal{P}}$-recognizable in $\mathcal{F}$ ) if every group $H \in \mathcal{F}$ with $\kappa_{\mathcal{P}}(H)=$ $\kappa_{\mathcal{P}}(G)$ is isomorphic to $G$. In other words, $G$ is $\kappa_{\mathcal{P}}$-recognizable in $\mathcal{F}$ if $h_{\mathcal{F}}(G)=1$, where $h_{\mathcal{F}}(G)$ is the (possibly infinite) number of pairwise non-isomorphic groups $H \in \mathcal{F}$ with $\kappa_{\mathcal{P}}(H)=\kappa_{\mathcal{P}}(G)$. We denote by $\mathcal{S}$ the classes of all finite simple groups. In the sequel, we show that the simple group $L_{2}(7) \cong L_{3}(2)$ is $\kappa_{\mathcal{P}}$-recognizable group in class $\mathcal{S}$, in other words $h_{\mathcal{S}}\left(L_{2}(7)\right)=1$.

Theorem 1. The simple group $L_{2}(7)$ is $\kappa_{\mathcal{P}}$-recognizable in the class $\mathcal{S}$ of all finite simple groups, that is, $h_{\mathcal{S}}\left(L_{2}(7)\right)=1$.

Proof. Let $G \in \mathcal{S}$ with $\kappa_{\mathcal{P}}(G)=\kappa_{\mathcal{P}}\left(L_{2}(7)\right)=2^{84} \cdot 3^{28} \cdot 7^{40}$ (see [33, Theorem 4.1]). We have to prove that $G$ is isomorphic to $L_{2}(7)$. Clearly, $G$ is nonabelian, since otherwise $G \cong \mathbb{Z}_{p}$ for some prime $p$, and so $\kappa_{\mathcal{P}}(G)=$ $\kappa_{\mathcal{P}}\left(\mathbb{Z}_{p}\right)=p^{p-2}$, which is a contradiction. Now, we claim that $\pi(G) \subseteq$ $\{2,3,5,7\}$. Suppose $p \in \pi(G)$ and $p \geqslant 11$. Let $c_{p}$ be the number of cyclic subgroups of order $p$ in $G$. By Lemma $6, c_{p} \geqslant p+1$, because $G$ is a nonabelian simple group. Therefore, from Lemma 5 , we deduce that

$$
\kappa_{\mathcal{P}}(G) \geqslant \kappa_{\mathcal{P}}\left(\mathbb{Z}_{p}\right)^{c_{p}} \geqslant \kappa_{\mathcal{P}}\left(\mathbb{Z}_{p}\right)^{p+1}=p^{(p-2)(p+1)} \geqslant 11^{108}>\kappa_{\mathcal{P}}(G),
$$

which is a contradiction. This shows that $\pi(G) \subseteq\{2,3,5,7\}$, as claimed. 
By results collected in [64, Table 1], $G$ is isomorphic to one of the groups $A_{5} \cong L_{2}(4) \cong L_{2}(5), A_{6} \cong L_{2}(9), S_{4}(3) \cong U_{4}(2), L_{2}(7) \cong L_{3}(2), L_{2}(8)$, $U_{3}(3), A_{7}, L_{2}(49), U_{3}(5), L_{3}(4), A_{8} \cong L_{4}(2), A_{9}, J_{2}, A_{10}, U_{4}(3), S_{4}(7), S_{6}(2)$ or $O_{8}^{+}(2)$. In all cases, except $A_{5}$ and $L_{2}(7), G$ contains a subgroup $H$ which is isomorphic to $A_{6}$ (see [19]). But then, $\kappa_{\mathcal{P}}(G) \geqslant \kappa_{\mathcal{P}}(H)=2^{180} \cdot 3^{40} \cdot 5^{108}$, a contradiction. If $G$ is isomorphic to $A_{5}$, then $\kappa_{\mathcal{P}}(G)=2^{20} \cdot 3^{10} \cdot 5^{18}$, which contradicts the assumption. Thus $G$ is isomorphic to $L_{2}(7)$, as required.

\subsection{Power-free decompositions}

A generalization of split graphs was introduced and investigated under the name $(m, n)$-graphs in [9]. A graph $\Gamma$ is an $(m, n)$-graph if its vertex set can be partitioned into $m$ cliques $C_{1}, \ldots, C_{m}$ and $n$ independent sets $I_{1}, \ldots, I_{n}$. In this situation,

$$
V_{\Gamma}=C_{1} \uplus C_{2} \uplus \cdots \uplus C_{m} \uplus I_{1} \uplus I_{2} \uplus \cdots \uplus I_{n},
$$

is called an $(m, n)$-split partition of $\Gamma$. Thus, $(m, n)$-graphs are a natural generalization of split graphs, which are precisely $(1,1)$-graphs.

Accordingly, we are motivated to make the following definition.

Definition 1. Let $G$ be a group and $n \geqslant 1$ an integer. We say that $G$ has an $n$-power-free decomposition if it can be partitioned as a disjoint union of a cyclic $p$-subgroup $C$ of maximal order and $n$ nonempty subsets $B_{1}, B_{2}, \ldots, B_{n}$ :

$$
G=C \uplus B_{1} \uplus B_{2} \uplus \cdots \uplus B_{n},
$$

such that the $B_{i}$ 's are independent sets in $\mathcal{P}(G)$ and $\left|B_{i}\right|>1$, for each $i$. If $n=1$, we simply say $G=C \uplus B_{1}$ is a power-free decomposition of $G$.

Since $C$ is a cyclic $p$-subgroup of maximal order in Definition $1, C$ is a clique, and so $\mathrm{Eq}(2)$ is a $(1, n)$-split partition of $\mathcal{P}(G)$. Note that, there are some finite groups which do not have an $n$-power-free decomposition, for any $n$, for example one can consider cyclic groups (see Proposition 1). On the other hand, the structure of groups $G$ which have a power-free decomposition is obtained (see Theorem 2).

Lemma 8. Suppose $G$ has an n-power-free decomposition:

$$
G=C \uplus B_{1} \uplus B_{2} \uplus \cdots \uplus B_{n},
$$

where $C$ is a cyclic p-subgroup of $G$. Then the following statements hold: 
(a) If $b \in G \backslash C$, then $\phi(o(b)) \leqslant n$. In particular, we have

$$
\pi(G) \subseteq \pi((n+1) !) \cup\{p\} .
$$

(b) If $p \notin \pi\left((n+1)\right.$ !), then $C$ is normal and $C_{C}(b)=1$ for each $b \in G \backslash C$. In particular, $Z(G)=1$.

(c) The set of universal vertices of $\mathcal{P}(G)$ is contained in $C$.

Proof. (a) The first statement follows immediately from the fact that the set of generators of cyclic group $\langle b\rangle$, which has $\phi(o(b))$ elements, forms a complete set in the $\mathcal{P}(G, G \backslash C)$, and hence each $B_{i}$ contains at most one of the generators. The second statement is also clear, because for each $q \in \pi(G) \backslash\{p\}$, there exists an element $b \in G \backslash C$ of order $q$, and so by first part $\phi(q)=q-1 \leqslant n$, or $q \leqslant n+1$.

(b) Assume the contrary. Let $C=\langle x\rangle$ with $o(x)=p^{m}>1$. Then, there exists $b \in G \backslash C$ such that $x^{b} \notin C$. By part (a) it follows that $\phi\left(o\left(x^{b}\right)\right) \leqslant n$. Since $o\left(x^{b}\right)=o(x)$,

$$
\phi\left(o\left(x^{b}\right)\right)=\phi(o(x))=\phi\left(p^{m}\right)=p^{m-1}(p-1),
$$

and so we obtain

$$
p-1 \leqslant p^{m-1}(p-1) \leqslant n .
$$

This forces $p \leqslant n+1$, which contradicts the hypothesis.

Let $b \in G \backslash C$. Suppose $c$ in $C$ is not the identity and commutes with $b$. Replacing $b$ by an appropriate power, we may assume without loss that $p$ divides $o(b c)$. Thus, we conclude that $p-1$ divides $\phi(o(b c))$. Since $b c \in G \backslash C$, by part (a) we have $\phi(o(b c)) \leqslant n$. Thus, it follows that $p-1 \leqslant n$, which is a contradiction. This shows that $C_{C}(b)=1$, as required.

(c) It is clear from Definition 1.

As the following result shows that there are some examples of groups for which there does not exist any $n$-power-free decomposition.

Proposition 1. Any cyclic group has no n-power-free decomposition.

Proof. Assume the contrary and let $G=\langle x\rangle$ be a cyclic group with an $n$-power-free decomposition:

$$
G=C \uplus B_{1} \uplus B_{2} \uplus \cdots \uplus B_{n},
$$


for some $n \geqslant 1$, where $C \subset G$ is a cyclic $p$-subgroup. Clearly, $x$ is a universal vertex in $\mathcal{P}(G)$, and so by Lemma 8 (c), $x \in C$. But then $C=G$, which is a contradiction. The proof is complete.

Proposition 2. The generalized quaternion group $Q_{2^{n}}, n \geqslant 3$, has a 2-power-free decomposition.

Proof. With the following presentation:

$$
Q_{2^{n}}=\left\langle x, y \mid x^{2^{n-1}}=1, y^{2}=x^{2^{n-2}}, x^{y}=x^{-1}\right\rangle,
$$

we may choose $C=\langle x\rangle$, and

$$
B_{1}=\left\{y, x y, \ldots, x^{2^{n-2}-1} y\right\}, B_{2}=\left\{x^{2^{n-2}} y, x^{2^{n-2}+1} y, \ldots, x^{2^{n-1}-1} y\right\} .
$$

Then $Q_{2^{n}}=C \uplus B_{1} \uplus B_{2}$ is a 2-power-free decomposition, and this completes the proof.

Given a group $G, 1 \in G$ is a universal vertex of the power graph $\mathcal{P}(G)$. Now, as an immediate corollary of Lemma 4 and Propositions 1 and 2, we have the following.

Corollary 2. Let $G$ be a group, $S$ the set of universal vertices of the power graph $\mathcal{P}(G)$, and $|S|>1$. Then $G$ has an n-power-free decomposition iff $G$ is isomorphic to a generalized quaternion group.

Theorem 2. The following conditions on a group $G$ are equivalent:

(a) $G$ has a power-free decomposition, $G=C \uplus B$, where $C$ is a cyclic p-subgroup of $G$.

(b) One of the following statements holds:

(1) $p=2$ and $G$ is an elementary abelian 2-group of order $\geqslant 4$.

(2) $p=2$ and $G$ is the dihedral group $D_{2^{m}}$ of order $2^{m}$, for some integer $m \geqslant 3$.

(3) $p>2$ and $G$ is the dihedral group $D_{2 p^{n}}$ (a Frobenius group) of order $2 p^{n}$ with a cyclic kernel of order $p^{n}$.

Proof. (a) $\Rightarrow$ (b). Suppose $G=C \uplus B$ is a power-free decomposition of $G$, where $C \subset G$ is a cyclic $p$-subgroup of maximal order. It follows by Lemma 8 (a) that every element $b \in B$ is an involution, and also $|G|=2^{m} p^{n}$, for some odd prime $p$ and $m \geqslant 1, n \geqslant 0$. We shall treat the cases $n=0$ and $n \geqslant 1$, separately.

Case 1. $n=0$. In this case, $G$ is a 2-group. If $|C| \leqslant 2$, then $G$ is an elementary abelian 2 -group and (1) holds. We may now assume that 
$|C|>2$. Put $C=\langle x\rangle$. Then, for every $b$ in $B, x^{b}$ is not an involution and so $x^{b} \in C$, which shows that $C$ is a normal subgroup of $G$. Thus $G / C$ is an elementary abelian 2-group by the previous paragraph.

We now claim that $[G: C]=2$. To prove this, we assume that $[G: C]=2^{t}$, where $t \geqslant 2$. Let $I=\operatorname{Inv}(G)$ be the set of involutions of $G$. Then, we have $I=B \cup\{z\}$, where $z$ is the unique involution in $C$, and so

$$
|I|=|B|+1=|G|-|C|+1=|G|-\frac{|G|}{2^{t}}+1=\left(\frac{2^{t}-1}{2^{t}}\right)|G|+1 \geqslant \frac{3}{4}|G|+1,
$$

which forces $G$ to be an abelian group. We recall that, a finite group is abelian if at least $3 / 4$ of its elements have order two. But then, if $b \in B$, then $b x$ is not an involution and also $b x \notin C$, which is a contradiction.

Let $b$ be an involution in $B$. Then $G=\langle x, b\rangle$. Since $b x \in B, b x$ is an involution, and thus $b x b=x^{-1}$, which implies that $G$ is a dihedral group and (2) follows.

Case 2. $n \geqslant 1$. In this case, $|G|=2^{m} p^{n}$ where $m, n \geqslant 1$, and $C$ is a cyclic $p$-group of maximal order. As in previous case $C=\langle x\rangle$ is a normal subgroup of $G$ and $G / C$ is an elementary abelian 2-group. Note that, $G$ does not contain an element of order $2 p$, and so $C=C_{G}(C)$. Moreover, since

$$
G / C=N_{G}(C) / C_{G}(C) \hookrightarrow \operatorname{Aut}(C),
$$

and $\operatorname{Aut}(C)$ is a cyclic group of order $\phi\left(p^{n}\right)=p^{n-1}(p-1)$, we conclude that $|G / C|=2$. Therefore, if $b$ is an involution in $G$, then

$$
G=\langle x, b\rangle=\langle x\rangle \rtimes\langle b\rangle,
$$

and since $b$ acts on $\langle x\rangle$ fixed-point-freely, $G$ is a Frobenius group of order $2 p^{n}$ with cyclic kernel $C$ of order $p^{n}$, and (3) follows.

(b) $\Rightarrow$ (a). Obviously.

\subsection{Commuting graphs}

In this section, we consider the problem of finding the tree-number of the commuting graphs associated with a family of finite simple groups. The Suzuki groups $\mathrm{Sz}(q)$, an infinite series of simple groups of Lie type, were defined in $[61,62]$ as subgroups of the groups $\mathrm{L}_{4}(q)$, with $q=2^{2 n+1} \geqslant 8$. In what follows, we shall give an explicit formula for $\kappa_{\mathcal{C}}(\operatorname{Sz}(q))$. Let $G=\operatorname{Sz}(q)$, where $q=2^{2 n+1}$. We begin with some well-known facts about the simple group $G$. These results have been obtained by Suzuki $[61,62]$ : 
(1) Let $r=2^{n+1}$. Then $|G|=q^{2}(q-1)\left(q^{2}+1\right)=q^{2}(q-1)(q-r+1)(q+$ $r+1)$, and $\mu(G)=\{4, q-1, q-r+1, q+r+1\}$. For convenience, we write $\alpha_{q}=q-r+1$ and $\beta_{q}=q+r+1$.

(2) Let $P$ be a Sylow 2-subgroup of $G$. Then $P$ is a 2-group of order $q^{2}$ with $\exp (P)=4$, which is a TI-subgroup, and $\left|N_{G}(P)\right|=q^{2}(q-1)$.

(3) Let $A \subset G$ be a cyclic subgroup of order $q-1$. Then $A$ is a TIsubgroup and the normalizer $N_{G}(A)$ is a dihedral group of order $2(q-1)$.

(4) Let $B \subset G$ be a cyclic subgroup of order $\alpha_{q}$. Then $B$ is a TI-subgroup and the normalizer $N_{G}(B)$ has order $4 \alpha_{q}$.

(5) Let $C \subset G$ be a cyclic subgroup of order $\beta_{q}$. Then $C$ is a TI-subgroup and the normalizer $N_{G}(C)$ has order $4 \beta_{q}$.

We recall that, in general, a subgroup $H \leqslant G$ is a TI-subgroup (trivial intersection subgroup) if for every $g \in G$, either $H^{g}=H$ or $H \cap H^{g}=\{1\}$.

Lemma 9. $\kappa_{\mathcal{C}}(P)=2^{(q-1)^{2}} q^{\left(q^{2}+q-3\right)}$.

Proof. By Theorem VIII.7.9 of [29] and Lemma XI.11.2 of [30], $Z(P)$ is an elementary abelian 2-group of order $q$ and every element outside $Z(P)$ has order 4 . Observe that $P$ is the centralizer in $G$ of all of the nontrivial elements of $Z(P)$. If $x \in P \backslash Z(P)$, then $\langle Z(P), x\rangle \leqslant C_{G}(x)$. In the proof of Lemma XI.11.7 of [30], we see that the elements of order 4 in $G$ lie in two conjugacy classes. This implies that $\left|C_{G}(x)\right|=2|Z(P)|$, from which we deduce that $C_{G}(x)=\langle Z(P), x\rangle$. Then for all $x, y \in P \backslash Z(P)$ either $C_{G}(x)=C_{G}(y)$ or $C_{G}(x) \cap C_{G}(y)=Z(P)$. Hence, $\left\{C_{G}(x) \mid x \in P \backslash Z(P)\right\}$ forms a partition of $P$ for which the intersection of pairwise centralizers is $Z(P)$. This shows that

$$
\mathcal{C}(P)=K_{q} \vee(\underbrace{K_{q} \oplus K_{q} \oplus \cdots \oplus K_{q}}_{q-1}) .
$$

Moreover, by Lemma 1, the eigenvalues of Laplacian matrix $L_{\mathcal{C}(P)}$ are:

$$
q^{2}, \underbrace{q^{2}, q^{2}, \ldots, q^{2}}_{q-1}, \underbrace{2 q, 2 q, \ldots, 2 q}_{(q-1)^{2}}, \underbrace{q, q, \ldots, q}_{q-2}, 0 .
$$

It follows immediately using Eq. (1) that

$$
\kappa_{\mathcal{C}}(P)=2^{(q-1)^{2}} q^{\left(q^{2}+q-3\right)},
$$

as required. 
Theorem 3. Let $q=2^{2 n+1}$, where $n \geqslant 1$ is an integer. Then, we have

$$
\kappa_{\mathcal{C}}(\mathrm{Sz}(q))=\left(2^{(q-1)^{2}} q^{\left(q^{2}+q-3\right)}\right)^{q^{2}+1}(q-1)^{(q-3) a}\left(\alpha_{q}\right)^{\left(\alpha_{q}-2\right) b}\left(\beta_{q}\right)^{\left(\beta_{q}-2\right) c},
$$

where $a=q^{2}\left(q^{2}+1\right) / 2, b=q^{2}(q-1) \beta_{q} / 4$ and $c=q^{2}(q-1) \alpha_{q} / 4$.

Proof. Let $G=\operatorname{Sz}(q)$, where $q=2^{2 n+1} \geqslant 8$. As already mentioned, $G$ contains a Sylow 2-subgroup $P$ of order $q^{2}$ and cyclic subgroups $A, B$, and $C$, of orders $q-1, \alpha_{q}$ and $\beta_{q}$, respectively. Moreover, every two distinct conjugates of them intersect trivially and every element of $G$ is a conjugate of an element in $P \cup A \cup B \cup C$. Looking at the proof of Lemma 11.6, we see that the cyclic subgroups $A, B$, and $C$, are the centralizers of their nonidentity elements, while $P$ is the centralizer in $G$ of all of the nontrivial elements of $Z(P)$. Let

$$
\begin{aligned}
G & =N_{P} x_{1} \cup \cdots \cup N_{P} x_{p}=N_{A} y_{1} \cup \cdots \cup N_{A} y_{a} \\
& =N_{B} z_{1} \cup \cdots \cup N_{B} z_{b}=N_{C} t_{1} \cup \cdots \cup N_{C} t_{c},
\end{aligned}
$$

be coset decompositions of $G$ by $N_{P}=N_{G}(P), N_{A}=N_{G}(A), N_{B}=$ $N_{G}(B)$ and $N_{C}=N_{G}(C)$, where $p=\left[G: N_{P}\right]=q^{2}+1, a=[G$ : $\left.N_{A}\right]=q^{2}\left(q^{2}+1\right) / 2, b=\left[G: N_{B}\right]=q^{2}(q-1) \beta_{q} / 4$ and $c=\left[G: N_{C}\right]=$ $q^{2}(q-1) \alpha_{q} / 4$. Then, we have

$G=P^{x_{1}} \cup \cdots \cup P^{x_{p}} \cup A^{y_{1}} \cup \cdots \cup A^{y_{a}} \cup B^{z_{1}} \cup \cdots \cup B^{z_{b}} \cup C^{t_{1}} \cup \cdots \cup C^{t_{c}}$.

This shows that

$$
\begin{aligned}
\mathcal{C}(G) & =K_{1} \vee\left(p \mathcal{C}\left(P^{\#}\right) \oplus a \mathcal{C}\left(A^{\#}\right) \oplus b \mathcal{C}\left(B^{\#}\right) \oplus c \mathcal{C}\left(C^{\#}\right)\right) \\
& =K_{1} \vee\left(p \mathcal{C}\left(P^{\#}\right) \oplus a K_{(q-1)-1} \oplus b K_{\alpha_{q}-1} \oplus c K_{\beta_{q}-1}\right),
\end{aligned}
$$

and so

$$
\kappa_{\mathcal{C}}(G)=\kappa_{\mathcal{C}}(P)^{p} \cdot \kappa_{\mathcal{C}}\left(K_{q-1}\right)^{a} \cdot \kappa_{\mathcal{C}}\left(K_{\alpha_{q}}\right)^{b} \cdot \kappa_{\mathcal{C}}\left(K_{\beta_{q}}\right)^{c} .
$$

Now, Lemma 9 and Cayley's formula yield the result.

\section{References}

[1] J. H. Abawajy, A. V. Kelarev and M. Chowdhury, Power graphs: a survey, Electron. J. Graph Theory Appl. (EJGTA), 1(2) (2013), 125-147.

[2] N. Akbari and A. R. Ashrafi, Note on the power graph of finite simple groups, Quasigroups Related Systems, 23(2) (2015), 165-173. 
[3] A. Ballester-Bolinches, J. Cossey and R. Esteban-Romero, A characterization via graphs of the soluble groups in which permutability is transitive, Algebra Discrete Math., 8(4)(2009), 10-17.

[4] S. Bera, On the intersection power graph of a finite group, Electron. J. Graph Theory Appl. (EJGTA), 6(1) (2018), 178-189.

[5] S. Bera and A. K. Bhuniya, On enhanced power graphs of finite groups, J. Algebra Appl., 17 (8)(2018), 1850146, 8 pp.

[6] A. K. Bhuniya and S. Bera, Normal subgroup based power graphs of a finite group, Comm. Algebra, 45(8) (2017), 3251-3259.

[7] A. K. Bhuniya and S. Bera, On some characterizations of strong power graphs of finite groups, Spec. Matrices, 4 (2016), 121-129.

[8] N. Biggs, Algebraic Graph Theory, Cambridge University Press, London, 1974.

[9] A. Brandstädt, Partitions of graphs into one or two independent sets and cliques, Discrete Math., 152(1-3) (1996), 47-54.

[10] J. R. Britnell and N. Gill, Perfect commuting graphs, J. Group Theory, 20(1) (2017), 71--102.

[11] D. Bubboloni, M. A. Iranmanesh, S. M. Shaker. Quotient graphs for power graphs, Rend. Semin. Mat. Univ. Padova, 138 (2017), 61-89.

[12] P. J. Cameron, The power graph of a finite group. II, J. Group Theory, 13(6) (2010), 779-783.

[13] P. J. Cameron, H. Guerra and S. Jurina, The power graph of a torsion-free group, J. Algebraic Combin., 49(1) (2019), 83-98.

[14] I. Chakrabarty, S. Ghosh and M. K. Sen, Undirected power graphs of semigroups, Semigroup Forum, 78 (2009), 410-426.

[15] S. Chattopadhyay and P. Panigrahi, Some structural properties of power graphs and $k$-power graphs of finite semigroups, J. Discrete Math. Sci. Cryptogr., 20(5) (2017), 1101-1119.

[16] S. Chattopadhyay and P. Panigrahi, Some relations between power graphs and Cayley graphs, J. Egyptian Math. Soc., 23(3) (2015), 457-462.

[17] S. Chattopadhyay and P. Panigrahi, Connectivity and planarity of power graphs of finite cyclic, dihedral and dicyclic groups, Algebra Discrete Math., 18(1) (2014), $42-49$.

[18] S. Chattopadhyay, P. Panigrahi and F. Atik, Spectral radius of power graphs on certain finite groups, Indag. Math. (N.S.), 29(2) (2018), 730-737.

[19] J. H. Conway, R. T. Curtis, S. P. Norton, R. A. Parker and R. A. Wilson R A, Atlas of Finite Groups, Oxford Clarendon Press, 1985.

[20] B. Curtin, G. R. Pourgholi and H. Yousefi-Azari, On the punctured power graph of a finite group, Australas. J. Combin., 62 (2015), 1-7.

[21] A. K. Das and D. Nongsiang, On the genus of the commuting graphs of finite nonabelian groups, Int. Electron. J. Algebra, 19 (2016), 91-109.

[22] A. Doostabadi and M. Farrokhi D. G., Embeddings of (proper) power graphs of finite groups, Algebra Discrete Math., 24(2) (2017), 221-234. 
[23] A. Doostabadi, M. Farrokhi D. Ghouchan, On the connectivity of proper power graphs of finite groups, Comm. Algebra, 43(10) (2015), 4305-4319.

[24] S. Földes and P. L. Hammer, Split graphs, Proceedings of the Eighth Southeastern Conference on Combinatorics, Graph Theory and Computing (Louisiana State Univ., Baton Rouge, La., 1977), 311-315.

[25] A. Hamzeh, Signless and normalized Laplacian spectrums of the power graph and its supergraphs of certain finite groups, J. Indones. Math. Soc., 24(1) (2018), 61-69.

[26] A. Hamzeh and A. R. Ashrafi,The order supergraph of the power graph of a finite group, Turkish J. Math., 42(4) (2018), 1978-1989.

[27] A. Hamzeh and A. R. Ashrafi, Spectrum and L-spectrum of the power graph and its main supergraph for certain finite groups, Filomat, 31(16) (2017), 5323-5334.

[28] A. Hamzeh and A. R. Ashrafi, Automorphism groups of supergraphs of the power graph of a finite group, European J. Combin., 60 (2017), 82-88.

[29] B. Huppert and N. Blackbrun, Finite Groups II, Springer-Verlag, Berlin, 1982.

[30] B. Huppert and N. Blackbrun, Finite Groups III, Springer-Verlag, Berlin, 1982.

[31] S. H. Jafari, Some results in a new power graphs in finite groups, Util. Math., 103 (2017), 181-187.

[32] S. H. Jafari, Some properties of power graphs in finite group, Asian-Eur. J. Math., 9 (4)(2016), 1650079, 6 pp.

[33] S. Kirkland, A. R. Moghaddamfar, S. Navid Salehy, S. Nima Salehy and M. Zohourattar, The complexity of power graphs associated with finite groups, Contributions to Discrete Mathematics, 13(2)(2018), 124-136.

[34] A. V. Kelarev, Graph Algebras and Automata, Marcel Dekker, New York, 2003.

[35] A. V. Kelarev, Ring Constructions and Applications, World Scientific, River Edge, NJ, 2002.

[36] A. V. Kelarev and S. J. Quinn, A combinatorial property and power graphs of groups, The Vienna Conference, Contrib. General Algebra, 12 (2000), 229-235.

[37] A. V. Kelarev and S. J. Quinn, Directed graphs and combinatorial properties of semigroups, J. Algebra, 251 (2002), 16-26.

[38] A. V. Kelarev and S. J. Quinn, A combinatorial property and power graphs of semigroups, Comment. Math. Univ. Carolinae, 45 (2004), 1-7.

[39] A. V. Kelarev, S. J. Quinn and R. Smolikova, Power graphs and semigroups of matrices, Bull. Austral. Math. Soc., 63 (2001), 341-344.

[40] A. Kelarev, J. Ryan, J. Yearwood, Cayley graphs as classifiers for data mining: The influence of asymmetries, Discrete Mathematics, 309 (2009), 5360-5369.

[41] X. Ma and M. Feng, On the chromatic number of the power graph of a finite group, Indag. Math. (N.S.), 26(4) (2015), 626-633.

[42] X. Ma, M. Feng and K. Wang, The rainbow connection number of the power graph of a finite group, Graphs Combin., 32(4) (2016), 1495-1504.

[43] X. Ma, R. Fu and X. Lu, On the independence number of the power graph of a finite group, Indag. Math. (N.S.), 29(2) (2018), 794-806. 
[44] X. Ma, R. Fu, X. Lu, M. Guoand Z. Zhao, Perfect codes in power graphs of finite groups, Open Math., 15 (2017), 1440-1449.

[45] A. Mahmoudifar and A. R. Moghaddamfar, Commuting graphs of groups and related numerical parameters, Comm. Algebra, 45(7)(2017), 3159-3165.

[46] S. K. Maity, Bipartite and planar power graphs of finite groups, Southeast Asian Bull. Math., 39(4) (2015), 539-543.

[47] Z. Mehranian, A. Gholami and A. R. Ashrafi, The spectra of power graphs of certain finite groups, Linear Multilinear Algebra, 65(5) (2017), 1003-1010.

[48] Z. Mehranian, A. Gholami and A. R. Ashrafi, A note on the power graph of a finite group, Int. J. Group Theory, 5(1) (2016), 1-10.

[49] R. Merris, Laplacian graph eigenvectors, Linear Algebra Appl., 278 (1998), 221-236.

[50] A. R. Moghaddamfar, S. Rahbariyan, S. Navid Salehy and S. Nima Salehy, The number of spanning trees of power graphs associated with specific groups and some applications, Ars Combinatoria, 113 (2017), 269-296.

[51] A. R. Moghaddamfar, S. Rahbariyan, and W. J. Shi, Certain properties of the power graph associated with a finite group, J. Algebra Appl., 13(7) (2014), 1450040, $18 \mathrm{pp}$.

[52] R. P. Panda and K. V. Krishna, On the minimum degree, edge-connectivity and connectivity of power graphs of finite groups, Comm. Algebra, 46(7) (2018), 3182-3197.

[53] R. P. Panda and K. V. Krishna, On connectedness of power graphs of finite groups, J. Algebra Appl., 17(10) (2018), 1850184, 20 pp.

[54] K. Pourghobadi and S. H. Jafari, The diameter of power graphs of symmetric groups, J. Algebra Appl., 17(12) (2018), 1850234, 11 pp.

[55] G. R. Pourgholi, H. Yousefi-Azari and A. R. Ashrafi, The undirected power graph of a finite group, Bull. Malays. Math. Sci. Soc., 38(4) (2015), 1517-1525.

[56] I. V. Protasov and K. D. Protasova, Automorphisms of kaleidoscopical graphs, Algebra Discrete Math., 6(2)(2007), 125-129.

[57] H. Sachs, On the number of spanning trees, Proceedings of the Fifth British Combinatorial Conference (Univ. Aberdeen, Aberdeen, 1975), pp. 529-535. Congressus Numerantium, No. XV, Utilitas Math., Winnipeg, Man., 1976.

[58] M. Shaker and M. A. Iranmanesh, On groups with specified quotient power graphs, Int. J. Group Theory, 5(3) (2016), 49-60.

[59] Y. Shitov, Coloring the power graph of a semigroup, Graphs Combin., 33(2) (2017), $485-487$.

[60] A. J. Slupik and V. I. Sushchansky, Minimal generating sets and Cayley graphs of Sylow p-subgroups of finite symmetric groups, Algebra Discrete Math., 8(4)(2009), 167-184.

[61] M. Suzuki, A new type of simple groups of finite order, Proc. Nat. Acad. Sci. U.S.A., 46 (1960), 868-870.

[62] M. Suzuki, On a class of doubly transitive groups, Ann. of Math., 75 (1) (1962), 105-145. 
[63] T. Tamizh Chelvam and M. Sattanathan, Power graph of finite abelian groups, Algebra Discrete Math., 16(1) (2013), 33-41.

[64] A. V. Zavarnitsine, Finite simple groups with narrow prime spectrum, Sib. Elektron. Mat. Izv., 6 (2009), 1-12.

\section{CONTACT INFORMATION}

Xiaoyou Chen

Ali Reza

Moghaddamfar, Mahsa Zohourattar
School of Sciences, Henan University of Technology, 450001, Zhengzhou, China E-Mail(s): cxy19800222@163.com

Faculty of Mathematics, K. N. Toosi University of Technology, P.O. Box 16315-1618, Tehran, Iran

E-Mail(s): moghadam@kntu.ac.ir, zohoorattar@mail.kntu.ac.ir Web-page(s): https:

//wp.kntu.ac.ir/moghadam/

Received by the editors: 06.06.2018

and in final form 04.03.2019. 